\title{
Near Real-Time Plaque Segmentation of IVUS
}

\author{
$\mathrm{O}_{\text {Pujol }}{ }^{1}, \mathrm{D}_{\text {Rotger }}{ }^{1}, \mathrm{P}_{\text {Radeva }}{ }^{1}, \mathrm{O}_{\text {Rodriguez }}{ }^{2}, \mathrm{~J} \mathrm{Mauri}^{2}$ \\ ${ }^{1}$ Computer Vision Center, UAB, Spain \\ ${ }^{2}$ Hospital Universitari Gmans. Tries i Pujol, Spain
}

\begin{abstract}
This article is devoted to the creation of a near-real time framework to discriminate between tissue and blood. We perform a fast supervised learning of local texture patterns of the plaque using Local Binary Patterns. A classifier is build by assembling weak classifiers using boosting schemes that allow quick performance and reliability. After that, a deformable model is used to ensure continuity in the segmentation and to fill in the gaps in the classification scheme. Our supervised learning framework has been validated using 450 test images from 15 different patients. The resulting segmentation differs from the physicians segmentation in a mean rate of $0.15 \mathrm{~mm}$. and maximum rate of $0.33 \mathrm{~mm}$. The method benefits from the low time consuming feature extraction, as well as a faster classification scheme reducing 10 times the whole processing time compared to most of the texture based approaches.
\end{abstract}

\section{Introduction}

Intravascular Ultrasound Images (IVUS) are a wellknown imaging technique for direct visualization of coronary arteries. However, visual evaluation and characterization of plaque require integration of complex information and suffer from substantial variability depending on the observer. This fact explains the difficulties of manual segmentation prone to high subjectivity in final results. Automatic segmentation will save time to physicians and provide objective vessel measurements. [1]

Nowadays, the most common methods to separate the tissue from the lumen are based on gray levels providing non-satisfactory segmentations. This leads to use more complex measures to discriminate lumen and plaque. One of the most wide spread methods in medical imaging for such task is texture analysis. The problem of texture analysis has played a prominent role in computer vision to solve problems of object segmentation and retrieval in numerous applications [2]. This approach, encodes the textural features of our image, and provide a feature space in which a classification based on such primitives is easier to perform.

Previous works in segmentation of IVUS images have shown different ways to segment lumen and to classify tissues [3], [4], [5]. However, these approaches usually are semi-automatic, sensitive to image artifacts and quite timeconsuming. In our approach we use Local Binary Patterns [6], which is a fast rotational invariant multi-resolution texture feature extractor based on "uniform" patterns since it is a fundamental property of texture.

The classification process is critical step in any image segmentation problem. Recently, arcing and boosting techniques have been applied successfully to different computer vision areas [7]. In this paper we analyze the relevance of boosting techniques, and in particular AdaBoost in Intravascular Ultrasound Image analysis. This process is integrated in an automatic framework for discrimination of lumen and plaque. The method is divided in 3 steps, corresponding to preprocessing step, feature extraction, classification, and higher level organization of data using deformable models. An objective evaluation of the different approaches is made and validated by the physicians in patients with different pathologies and images with different topologies.

The paper is organized as follows: section 2 describes the Local Binary Pattern features; section 3 introduces the AdaBoost procedure for feature selection and classification assembling of "weak" classifiers; section 4 presents a concise description of snakes; section 5 shows the results of the methods and section 6 discuss the future lines.

\section{Local Binary Patterns for feature extraction}

Local Binary Patterns is a feature extraction operator used for detecting "uniform" local binary patterns at circular neighborhoods of any quantization of the angular space and at any spatial resolution. The operator is derived based on a circularly symmetric neighbor set of $P$ members on a circle of radius $R$. The operator is denoted by $L B P_{P, R}^{r i u 2}$. Parameter $P$ controls the quantization of the angular space, and $R$ determines the spatial resolution of the operator. Figure 1 shows typical neighbors sets. To achieve gray-scale invariance, the gray value of the center 

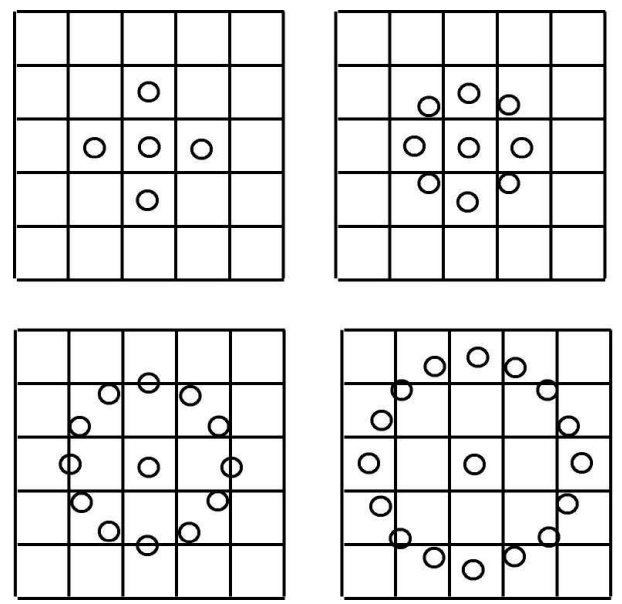

Figure 1. Typical neighbors (Top-Left) $P=4, R=1.0$ (Top-Right) $P=8, R=1.0$ (Bottom-Left) $P=12, R=$ 1.5 (Bottom-Right) $P=16, R=2.0$.

pixel $\left(g_{c}\right)$ is subtracted from the gray values of the circularly symmetric neighborhood $g_{p}(p=0,1, \ldots, P-1)$ and assigned a 1 value if the difference is positive and 0 if negative.

$$
s(x)= \begin{cases}1 & \text { if } x \geq 0 \\ 0 & \text { otherwise }\end{cases}
$$

By assigning a binomial factor $2^{p}$ for each value obtained we transform the neighborhood into a single value. this value is the $L B P_{R, P}$

$$
L B P_{R, P}=\sum_{p=0}^{P} s\left(g_{p}-g_{c}\right) \cdot 2^{p}
$$

To achieve rotation invariance the pattern set is rotated as many times as necessary to achieve a maximal number of the most significant bits, starting always from the same pixel. The last stage of the operator consist on keep the information of "uniform" patterns while filtering the rest. This is achieved using a transition count function $U$. $U$ is a function which counts the number of transitions $0 / 1,1 / 0$ while we move over the neighborhood.

$$
\begin{aligned}
U\left(L B P_{P, R}\right)= & \left|s\left(g_{P-1}-g_{c}\right)-s\left(g_{0}-g_{c}\right)\right|+ \\
& \sum_{p=1}^{P-1}\left|s\left(g_{p}-g_{c}\right)-s\left(g_{p-1}-g_{c}\right)\right|
\end{aligned}
$$

therefore

$$
L B P_{P, R}^{r i u 2}=\left\{\begin{array}{cl}
L B P_{P, R}^{r i} & \text { if } U\left(L B P_{P, R}\right) \leq 2 \\
P+1 & \text { otherwise }
\end{array}\right.
$$

The resulting operator is a powerful tool for texture discrimination, and in this paper we prove that it is a good feature extractor for Intravascular Ultrasound Image segmentation.

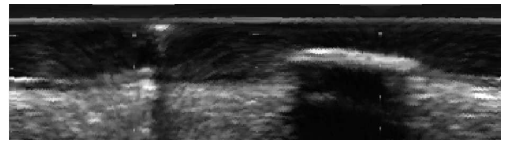

(a)

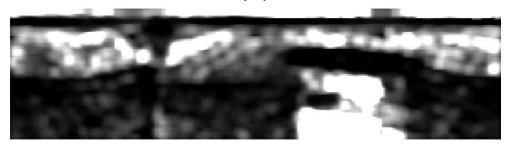

(b)

Figure 2. Local Binary Pattern response. (a) Original image. (b) Local Binary Pattern output with parameters $R=3, P=24$.

\section{AdaBoost for classification}

High performing and fastness are two of the main features desired for a classifier. However, they are usually mutually excluding, so we can find fast, low timeconsuming classifiers but with relative bad performance. Nevertheless, on the last few years, the subject of how to take advantage of the fast classifiers by assembling has been studied. This study has led to boosting and arcing procedures. Recently, arcing procedures (Adaptative reweighing and combining) have been credited to lead to very high classification ratios using "weak" learning processes [7], [8]. Boosting allows the use of "weak" classifiers with accuracy on the training set greater random classification. The goal is to create a high performing classifier ensemble of "weak" classifiers.

Adaptative Boosting (AdaBoost) is an arcing method which allows the designer to continue adding "weak" classifiers until some desired low training error has been achieved. A weight is assigned to each of the feature points. These weights measure how accurate the feature point is being classified. If it is accurately classified, then its probability of being used in subsequent learners is reduced, or emphasized otherwise. This way, AdaBoost focuses on difficult features.

In our problem, the different classes are tissue and blood. For each feature, the weak learner determines the optimal classification threshold function, so that the minimum number of feature points is misclassified. The algorithm is described as follows

- Determine a supervised set of feature points $\left\{x_{i}, c_{i}\right\}$ where $c_{i}=\{-1,1\}$ is the class associated to each of the features classes (blood and tissue respectively)

- Initialize weights $w_{1, i}=\frac{1}{2 m}, \frac{1}{2 l}$ for $c_{i}=\{-1,1\}$ respectively, where $m$ and $l$ are the number of feature points for each class.

- For $t=1 . . T$ : 
- Normalize weights

$$
w_{t, i} \leftarrow \frac{w_{t, i}}{\sum_{j=1}^{n} w_{t, i}}
$$

so that $w_{t}$ is a probability distribution.

- Train a classifier, $h$. The error is evaluated with respect to $w_{t}, \epsilon_{j}=\sum_{i} w_{i}\left|h\left(x_{i}\right)-c_{i}\right|$.

- Update the weights:

$$
w_{t+1, i}=w_{t, i} \beta_{t}^{e_{i}}
$$

where $e_{i}=1$ for each well-classified feature and $e_{i}=0$ otherwise. $\beta_{t}=\frac{\epsilon_{t}}{1-\epsilon_{t}}$. Calculate parameter $\alpha_{t}=-\log \left(\beta_{t}\right)$.

- The final "strong" classifier is:

$$
h(x)= \begin{cases}1 & \sum_{t=1}^{T} \alpha_{t} h_{t}(x) \geq 0 \\ 0 & \text { otherwise }\end{cases}
$$

Therefore, the strong classifier is the ensemble of a series of simple classifiers ("weak"). Parameter $\alpha_{t}$ is the weighting factor of each of the classifiers. The loop ends whether the classification error of a "weak" classifier is over 0.5 , the estimated error for the whole "strong" classifier is lower than a given error rate or if we achieve the desired number of "weaks".

The weak classifier has an important role in the procedure. Different approaches can be used, however it is relatively interesting to center our attention in low timeconsuming classifiers. We have chosen a simple approach by modelling the feature points as Gaussian distributions. This scheme allows us to introduce the weights easily, simply by calculating the weighed mean and covariance of the classes at each step of the process:

$$
\mu_{i, t}^{j}=\sum w_{i, t} x_{i} \quad \Sigma_{i, t}^{j}=\sum w_{i, t}\left(x_{i}-\mu_{i, t}^{j}\right)^{2}
$$

for each $x_{i}^{j}$ point in class $C_{j} . W_{i, j}$ are the weights for each data point.

\section{Snake-based accurate location of borders}

The result after the classification step is a clear image in which the main structures are clearly visible, but there is no connection among them. This is a good example in which a snake can be useful. The basic target of active contours [9] [10] is to find a parameterized curve that minimizes the weighted sum of its internal energy and external energy. Given a traditional snake curve $\mathbf{x}(s)=(x(s), y(s)), s \in$ $[0,1]$, the snake can be formulated as the minimization of the equation $S(\mathbf{x})=\int_{0}^{1}\left(\alpha\left|\mathbf{x}^{\prime}(s)\right|^{2}+\beta\left|\mathbf{x}^{\prime \prime}(s)\right|+E_{e}\right) d s$;
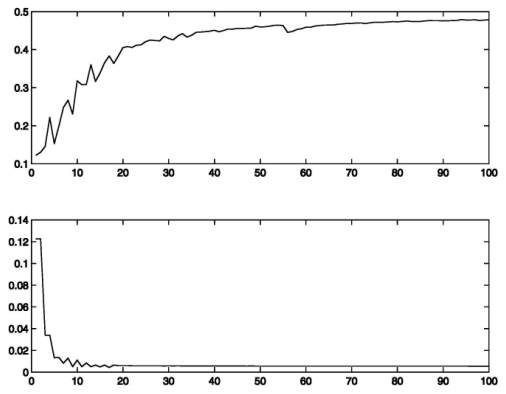

(a)

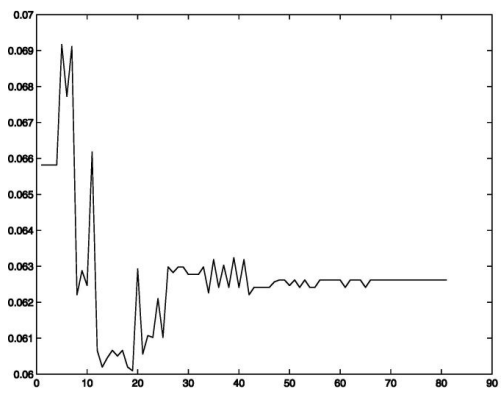

(b)

Figure 3. Training error of the process for a mixed set of feature spaces. (a) (top) Weak classification error. (bottom) Strong classification error. (b) Test classification error.

where $\alpha$ and $\beta$ are weighting factors and $E_{e}$ the external energy.

The typical potential function designed to lead a deformable contour toward step edges is $P(x, y)=$ $-\gamma\left|\nabla\left[G_{\sigma}(x, y) * I(x, y)\right]\right|^{2}$ where $\nabla$ is the gradient operator, $\gamma$ is a weighting parameter, $G_{\sigma}(x, y)$ is the gaussian filter of standard deviation $\sigma$, and $I(x, y)$ is the image data. As can be observed greater $\sigma$ will increase the attraction range but the edges will blur. In our experiments the snake is initialized at the top row of the image and is attracted by the classification edges; therefore $I(x, y)$ will be the resulting classification image.

\section{Experimental results}

We have used images from 5 different patients to build the training set. Segmentation of those images in the training step has been manually guided by experts. The test set is composed by different images of 10 different patients. Feature extraction is done using Local Binary Patterns with $P=8,16,24$ and $R=1,2,3$. As local binary patterns are insensible to contrast we introduced the local variance using the same neighborhoods as in LBP. Figure 2 shows the output of the local binary pattern operator with parameters $R=3, P=24$. As can be seen in the figure, the tissue has lower values than the blood structures. On the other hand, shadowed areas of the intravascular image has really high 


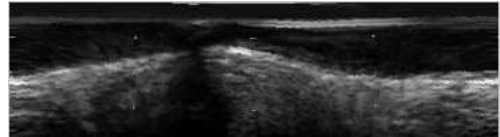

(a)

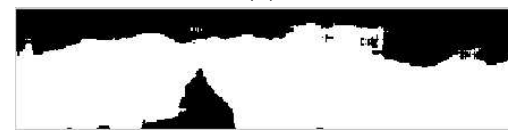

(b)

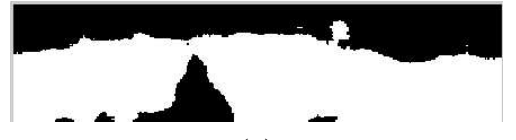

(c)

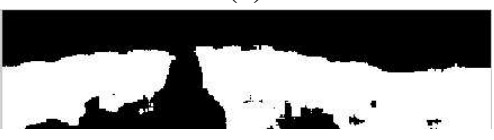

(d)

Figure 4. Test classification images at different stages of the strong classifier. (a) Original image (b) "Strong" with 3 "weaks" (c) "Strong" with 7 "weaks" (d) "Strong" with 22 "weaks"

values in the feature space.

Figure 3 shows the typical behavior of the training process of the AdaBoost classifier for a mixed set of feature spaces and a simple "weak" classifier approach. Figure 3.a.(top) shows the error rate of each of the "weak" classifiers. Each time a "weak" is assembled, feature are more difficult to classify due to the weights influence, therefore the "weak" classification error increases. However, 3.a.(bottom) shows how the joint error of the ensemble of the "weak" classifiers (the "strong" classifier) decreases as more "weaks" are assembled. Figure 3.b illustrates the test error.

Figure 4 shows the evolution of the classification image. It can be seen that the classification tends to be better the most classifiers are assembled. However it must be said that most of the time it tends to overfit or to stabilize in a fixed error rate; hence, the number of the reliable "weak" classifiers for the application must be found. This can be done using cross-validation processes.

We have applied this fully automatic integration framework described in the former section to different sequences from 5 patients, for validation of the methodology. The resulting segmentation differs from the physicians segmentation in a mean rate of $0.15 \mathrm{~mm}$. and maximum rate of $0.33 \mathrm{~mm}$. This error rates are comparable to the detection schemes found in literature. However, the classification step is performed in very little time, allowing this kind of schemes apt for real-time detection processes.

\section{Conclusion and future lines}

We have presented an integration framework for tissueblood segmentation in IVUS images using a classifier ensemble with Adaboost over the Local Binary Pattern features. The framework composed by LBP - Adaboost - Snakes is strong and fast performing, leading to very accurate results. Our current works aim for a near real-time tissue characterization, which can be achieved without increasing the processing time by using the same framework since Local Binary Patterns features provide enough information for tissue discrimination.

\section{Acknowledgements}

This work was partially supported by the project TIC2000-1635-C04-04 of CICYT, Ministerio de Ciencia y Tecnologa of Spain.

\section{References}

[1] Sonka M, Zhang X, Siebes M. Segmentation of intravascular ultrasound images: A knowledge based approach. IEEE Trans. on Medical Imaging, 1995;14:719-32.

[2] Malik J, Belongie S, Leung T, Shi J. Contour and Texture Analysis for Image Segmentation. International Journal of Computer Vision, 2001;43:7-27.

[3] Zhang X, Sonka M. Tissue Characterization in intravascular ultrasound images. IEEE Trans. on Medical Imaging, 1998;17:889-99.

[4] von Birgelen C. Computerized assessment of coronary lumen and atherosclerotic plaque dimensions in threedimensional intravascular ultrasound correlated with histomorphometry. Amer. J. Cardiol, 1996;78:1202-9.

[5] Klingensmith JD, Shekhar R, Vince DG. Evaluation of Three-Dimensional Segmentation Algorithms for Identification of Luminal and Medial-Adventitial Borders in Intravascular Ultrasound Images. IEEE Trans. on Medical Imaging, 2000;19:996-1011.

[6] Ojala T, Pietikainen M, Maenpaa T. Multiresolution GrayScale and Rotation Invariant Texture Classification with Local Binary Patterns. IEEE Trans. on Pat. Anal. and Mach. Int, 2002;24:971-87.

[7] Schapire RE. The Boosting Approach to Machine Learning. An Overview. MSRI Workshop on Nonlinear Estimation and Classification. 2002.

[8] Viola P, Jones M. Rapid object detection using a boosted cascade of simple features. Conference on Computer Vision and Pattern Recognition, 2001:511-18.

[9] Kass M, Witkin A, Terzopoulos D. Snakes, Active contour models. Int. J. Computer Vision, 1987;1:321-331.

[10] McInerney T, Terzopoulos D. Deformable models in medical images analysis: a survey. Medical Image Analysis, 1996:1:91-108. 